\title{
Individuelle Unterschiede bei der Behandlung von Plasmen und Seren mit $\beta$-Propiolacton
}

\author{
Von W. STEPHAN \\ Aus der Wissenscbaftlichen Abteilung der Biotest-Serum-Institut GmbH, Frankfurt|Main \\ (Leiter: Privatdozent Dr. H. Determann)
}

(Eingegangen am 22. Juli 1968)

Die Modifizierung von Proteinen in Plasmen und Seren durch $\beta$-Propiolacton hängt von der Hydrolysegeschwindigkeit des $\beta$-Propiolactons in in Plasmen bzw. Seren ab: geringe Hydrolysegeschwindigkeit bewirkt starke Modifizierung.

Es wurde gefunden, daß die Hydrolysegeschwindigkeit von $\beta$-Propiolacton in Plasmen und Seren nicht konstant, sondern spenderabhängig ist. Dies hat zur Folge, daß nur größere Plasma- und Serum-Pools ohne Gefahr der Modifizierung mit $\beta$-Propiolacton behandelt werden können, während die $\beta$-Propiolacton-Behandlung von Einzelspenderplasmen und -seren gegebenenfalls zu starken Proteinmodifizierungen führt.

\section{Differences between individual plasmas and sera on treatment with $\beta$-propiolactone}

The extent to which the proteins of plasmas and sera are modified by $\beta$-propiolactone depends on the rate of hydrolysis of the $\beta$-propiolactone by the plasmas and sera: if the rate of hydrolysis is low, the degree of modification is high.

It was found that the rate of hydrolysis of $\beta$-propiolactone by plasmas and sera is not uniform, but depends upon the donor. This means that only large plasma and serum pools can be treated with $\beta$-propiolactone without danger of modification, while the treatment of single donor samples causes marked modification of the proteins.

Logrippo empfiehlt zur Entfernung von HepatitisViren aus Plasmapools die Kombination von $\beta$-Propiolacton-Behandlung $(0,3 \mathrm{~g} / 100 \mathrm{ml})$ und UV-Bestrahlung (1). Eine Modifizierung der Plasmaproteine tritt unter den angegebenen Bedingungen nicht ein (2). Nach unseren Erfahrungen werden Proteinmodifizierungen dagegen dann beobachtet, wenin vor $\operatorname{der} \beta$ Propiolacton-Behandlung eine Adsorption, zum Beispiel mit Aerosil, stattgefunden hat (3).

Im Zusammenhang mit dem von uns untersuchten Adsorptionsprozeß wurde eine $\beta$-Propiolacton-spaltende Hydrolase (Esterase) diskutiert, die bei der Adsorption aus dem Plasma bzw. Serum entfernt wird: weil das $\beta$-Propiolacton nun langsamer hydrolysiert wird, steht es in größerem Umfang für die Reaktion mit den $\mathrm{NH}_{2}$-Gruppen der Proteine zur Verfügung. Dies bedingt eine Abnahme der basischen Gruppen und damit eine erhöhte elektrophoretische Wanderungsgeschwindigkeit der Proteine in alkalischen Puffern.

Es wurde nun gefunden, daß die Hydrolysegeschwindigkeit von $\beta$-Propiolacton in Plasmen und Seren verschiedener menschlicher Individuen eine unterschiedliche Größe hat.

Hydrolyse von $\beta$-Propiolacton in Einzelspender-Plasmen und -Seren

Die Unterschiede in der Hydrolysegeschwindigkeit von $\beta$-Propiolacton in Einzelspender-Plasmen und -Seren sind signifikant, wie sich aus Tabelle 1 ergibt.

Die laufende Überwachung einzelner Spender zeigte, $\mathrm{da} \beta$ die Hydrolysegeschwindigkeit von $\beta$-Propiolacton in deren Seren innerhalb eines längeren Zeitraumes konstant ist.

Diese Konstanz ist ein Hinweis darauf, daß die Hydrolyseaktivität eine individuelle Größe ist. Die weiteren Versuche zeigten, daß die $\beta$-Propiolacton-Behandlung bei „aktiven" Plasmen und Seren (schnelle Hydrolyse) und ,inaktiven" Plasmen und Seren (langsame Hydrolyse) bezüglich der Modifizierung der Plasmafraktionen völlig verschiedene Resultate liefert.

Proteinmodifizierung durch $\beta$-Propiolacton in Abhängigkeit der Hydrolysegeschwindigkeit von $\beta$-Propiolacton in Plasmen und Seren

Während die Proteine von „aktiven“ Einzel-Plasmen und -Seren nach $\beta$-Propiolacton-Behandlung immunelektrophoretisch keine nachweisbaren Veränderungen

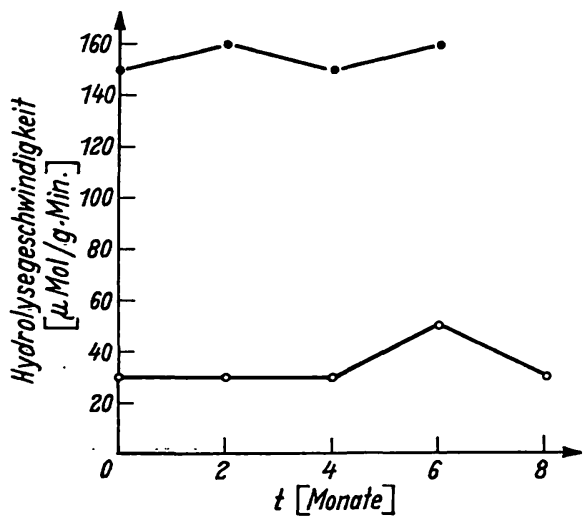

Abb. 1

Konstanz der $\beta$-Propiolacton-Hydrolyse in Seren über längere Zeit. - aktives Serum c inaktives Serum

Tab. 1

Hydrolysegeschwindigkeit von $\dot{\beta}$-Propiolacton $(\mu \mathrm{Mol} / \mathrm{g} \cdot \mathrm{Min}$.$) in verschiedenen Einzelspender-Plasmen und -$ Seren

\begin{tabular}{|c|c|c|c|c|c|c|c|c|c|c|c|}
\hline Laufende Nr. & 1 & 2 & 3 & 4 & 5 & 6 & 7 & 8 & 9 & 10 & 11 \\
\hline $\begin{array}{l}\text { Hydrolysegeschwindigkeit in Serum } \\
\text { Hydrolysegeschwindigkeit in Plasma }\end{array}$ & $\begin{array}{r}170 \\
80\end{array}$ & $\begin{array}{r}105 \\
20\end{array}$ & $\begin{array}{l}95 \\
60\end{array}$ & $\begin{array}{r}135 \\
65\end{array}$ & $\begin{array}{l}50 \\
35\end{array}$ & $\begin{array}{r}50 \\
150\end{array}$ & $\begin{array}{r}110 \\
30\end{array}$ & $\begin{array}{l}30 \\
15\end{array}$ & $\begin{array}{l}125 \\
120\end{array}$ & $\begin{array}{l}45 \\
30\end{array}$ & $\begin{array}{r}150 \\
95\end{array}$ \\
\hline
\end{tabular}

Das unter der gleichen laufenden Nummer aufgẹührtẹ Plasma und Serum stammt nicht vom selben Spender. 
erkennen lassen, werden die Proteine ,inaktiver" Plasmen und Seren unter den gleichen Bedingungen durch $0,3 \mathrm{~g} / 100 \mathrm{~m} / \beta$-Propiolacton so stark modifiziert, $\mathrm{da} B$ eine deutliche Erhöhung der elektrophoretischen Wanderungsgeschwindigkeit in alkalischen Puffern nachweisbar ist.

Aus Abbildung 2 erkennt man, da $\beta$ die Fraktionen des mit $\beta$-Propiolacton behandelten (,inaktiven“) Plasmas 2 wesentlich rascher wandern als die des mit $\beta$-Propiolacton behandelten (,aktiven“) Plasmas 1.

Um zu zeigen, daß hier eine chemische Modifizierung vorliegt und nicht etwa eine Anlagerung von $\beta$-Hydroxypropionsäure, wurde das stark modifizierte Plasma mit dem nicht modifizierten Ausgangsplasma gemischt und 2 Tage bei $37^{\circ}$ inkubiert (um gegebenenfalls einen Ionenübergang zu ermöglichen). Die Mischung wurde dann immunelektrophoretisch untersucht.

Die chemische Modifizierung gibt sich durch das Vorliegen von 2 Arten von Plasmaproteinen zu erkennen: schnell und langsam wandernde, deren Präzipitationsbögen ohne Spornbildung ineinander übergehen.

Bei Verwendung von Anti-Humanserum vom Pferd (Abb. 3) ist die immunologische Identität der modifizierten und nicht modifizierten Globuline infolge der scharfen Präzipitationsbögen besonders gut zu erkennen, während die Darstellung mit Anti-Humanserum vom Kaninchen (Abb. 4) die Veränderung des Albumins besonders deutlich macht. Außerdem sieht man, daß nicht alle Proteine gleich stark modifiziert werden: Albumin und Transferrin reagieren besonders stark mit $\beta$-Propiolacton, während das $\alpha_{2}$-Lipoprotein kaum verändert wird.

Die modifizierten Proteine werden auch in ihren spezifischen Aktivitäten verändert, wie gerinnungsanalytische Untersuchungen zeigen. Hier wurde gefunden, $\mathrm{da} \beta$ nach Behandlung eines ,inaktiven" Plasmas mit $0,3 \mathrm{~g} / 100 \mathrm{~m} l \beta$-Propiolacton dieses Plasma auf Zusatz von Thrombin nicht mehr gerinnt, während die $\beta$ Propiolacton-Behandlung eines "aktiven“ Plasmas die Gerinnung nicht beeinträchtigt (Tab. 2).

Diese Unterschiede verschwinden bei $\operatorname{der} \beta$-Propiolacton-Behandlung großer Plasma- oder Serumpools. In ihnen werden die in der Minderzahl vorhandenen „inaktiven“ Plasmen bzw. Seren durch besonders "aktive“ kompensiert, so daß eine genügend hohe Hydrolysegeschwindigkeit resultiert. Daher treten bei Anwendung der von Lo GrIPpo vorgeschlagenen Dosis von $0,3 \mathrm{~g} / 100 \mathrm{~m} l \beta$-Propiolacton keine Proteinmodifizierungen auf.

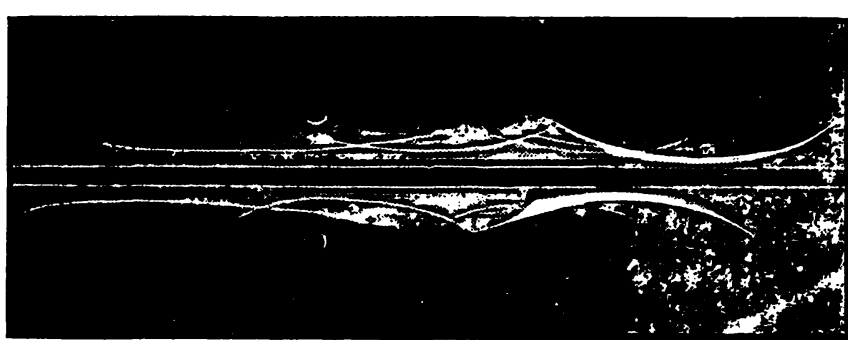

Abb. 2

Immunelektrophoretische Auftrennung von $\beta$-Propiolacton-behandelten Plasmen, oben: , inaktives“" Plasma (Nr. 2), unten: , aktives" Plasma (Nr. 1). Darstellung mit Anti-Humanserum von Kaninchen

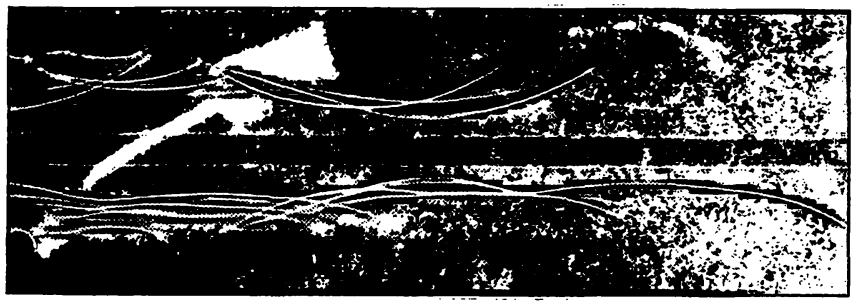

Abb. 3

Immunelektrophoretische Auftrennung der Mischung aus $\beta$-Propiolacton-modifiziertem, ,inaktivem" Plasma und nicht modifiziertem Ausgangsplasma (unten). Das Ausgangsplasma ist oben aufgetragen. Darstellung mit Anti-Humanserum vom Pferd

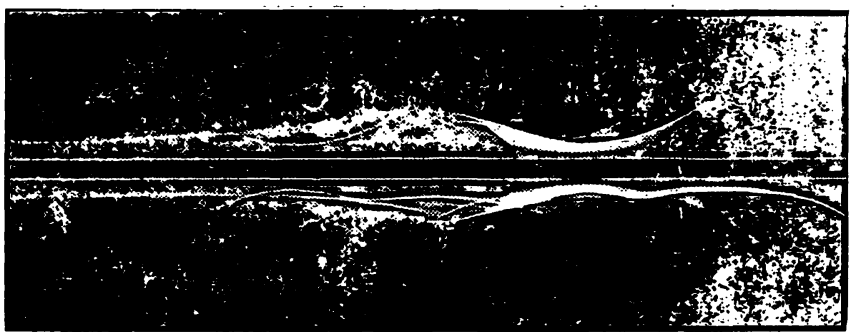

Abb. 4

Immunelektrophoretische Auftrennung der Mischung aus $\beta$-Propiolacton-modifiziertem ,inaktivem" Plasma und nicht modifiziertem Ausgangsplasma (unten). Das Ausgangsplasma ist oben aufgetragen. Darstellung mit Anti-Humanserum vom Kaninchen

\section{Beschreibung der Versuche}

\section{Messung der $\beta$-Propiolacton-Hydrolyse}

Die Bestimmung wurde mit einer Kombination von Titrigraph, Titrator und Autobürette der Fa. Radiometer, Kopenhagen, durchgeführt. Die Eichung erfolgte mit Puffertitrisol pH 8,0 bei $20^{\circ}$.

$20 \mathrm{~m} l$ der zu testenden Lösung (z. B. $5 \mathrm{~m} l$ Serum $+15 \mathrm{~m} l$ physiol. $\mathrm{NaCl}-\mathrm{Lösung}$ ) werden vorgelegt und auf $\mathrm{pH} 8,0$ eingestellt. Dann werden $0,125 \mathrm{~m} l \beta$-Propiolacton ( $=2 \mathrm{mMol}$ ) zugefügt und mit $2 \mathrm{~m} l$ physiol. NaCl-Lösung nachgespült. Man titriert mit $1 \mathrm{NNaOH}$ $10 \mathrm{Min}$. und registriert den $\mathrm{NaOH}-$ Verbrauch pro Zeit. Aus dem durchschnittlichen Verbrauch an $1 \mathrm{~N} \mathrm{NaOH}$ in der Anfangsphase der Hydrolyse, bezogen auf $1 \mathrm{~g}$ Protein und $1 \mathrm{Min}$., ergibt sich die spezifische Aktivität des entsprechenden Serums bzw. Plasmas bezüglich der Hydrolyse von $\beta$-Propiolacton, ausgedrückt in $\mu \mathrm{Mol} / \mathrm{g} \cdot \mathrm{Min}$.

Tab. 2

Zusammenhang zwischen Gerinnung und $\beta$-Propiolâcton-Behandlung bei verschiedenen Plasmen

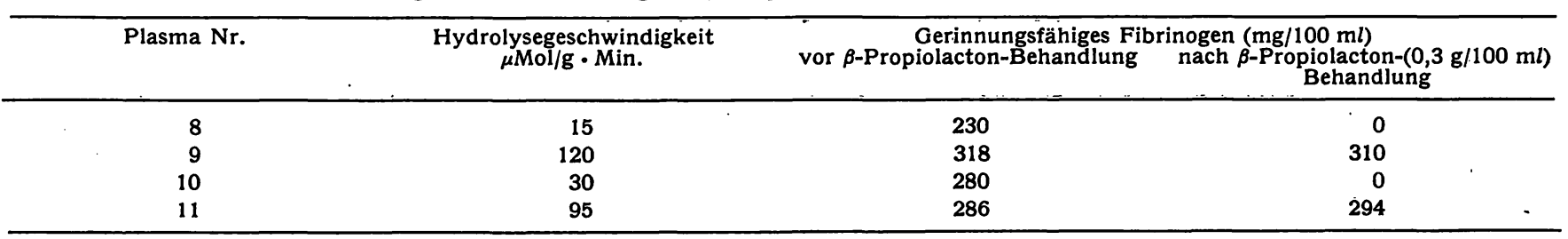




\section{$\beta$-Propiolacton-Bebandlung}

Das zu behandelnde Serum wird auf $5^{\circ}$ abgekühlt und $0,3 \mathrm{~g}$ $\beta$-Propiolacton pro $100 \mathrm{~m} l$ Serum zugegeben. Man läßt 1 Std. bei $5^{\circ}$ stehen, stellt den $\mathrm{pH}-\mathrm{Wert}$ auf $8,0 \mathrm{mit} 1 \mathrm{~N} \mathrm{NaOH}$ ein und hydrolysiert das restliche $\beta$-Propiolacton bei $37^{\circ}$ im pH-Stat bei $\mathrm{pH} 8,0$ während etwa 2-4 Stdn. Analoges gilt für Plasma.

\section{Immunelektroplsorese}

Es wurden folgende Antiseren verwendet:

Antihumanserum vom Kaninchen (Behringwerke, Marburg), Antihumanserum vom Pferd (Biotest-Serum-Institut, Frankfurt).

Die Menge an Antiserum betrug zwischen $0,02 \mathrm{ml}$ und $0,04 \mathrm{ml}$.

4. Fibrinogen-Bestimmung

Reagenzien: $35 \mathrm{ml} 0,9$ proz. $\mathrm{NaCl}$

$1 \mathrm{ml} 25 \mathrm{mM} \mathrm{CaCl}$-Lösung

$0,5 \mathrm{ml} 0,1 \mathrm{proz}$. wäßr. Thrombinlösung.
$1,0 \mathrm{ml}$ Plasma wird in ein $50 \mathrm{~m} /$-Zentrifugenglas einpipettiert, mit den angegebenen Mengen Reagenzien zur Durchmischung leicht bewegt und $1 \mathrm{Std}$. im Wasserbad bei $37^{\circ}$ inkubiert. Anschließend wird $20 \mathrm{Min}$. bei $5000 \mathrm{U}$./Min. zentrifugiert. Das am Boden des Glases zusammengeballte Gerinnsel wird mit einem PVC-Stäbchen sauber herausgefischt und auf einem Filtrierpapier getrocknet. Das ausgedrückte Gerinnsel wird mit Wasser abgespült und nochmals auf Filtrierpapier abgepreßt.

$\mathrm{Zu}$ dem in ein Wassermannröhrchen abgestreiften Gerinnsel werden $2,5 \mathrm{ml} 10$ proz. $\mathrm{NaOH}$ hinzupipettiert. Zur vollständigen Auflösung wird $30 \mathrm{Min}$. bei $37^{\circ}$ inkubiert. Nach Zusatz von $2,5 \mathrm{~m} l$ Biuret-Lösung wird der Proteingehalt gegen eine Mischung aus 10proz. $\mathrm{NaOH}$ und Biuretlösung im Verhältnis 1:1 bestimmt.

Frl. E. Haug und Frl. E. Fries danke ich für die Durchführung der Versuche.

\section{Literatur}

1. Lo Grippo, G. A., in „Hepatitis Prontiers“, 499. Henry Ford Hospital International Symposium Churchill Ltd., London (1957).
2. Lo Grippo, G. A., Ann. N. Y. Acad. Sci. 83, 578 (1960). 3. STEPHAN, W. und L. RóKA, diese Z. 6, 186 (1968).
Dr. W. Stephan Biotest-Serum Institut 6000 Frankfurt/Main Flughafenstr. 4 